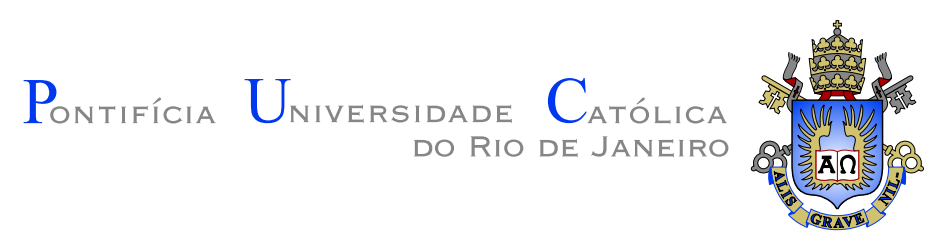

Baldoino Fonsêca dos Santos Neto

\title{
Uma Abordagem Deontica para o Desenvolvimento de Agentes Normativos Autônomos
}

Tese apresentada ao Programa de Pós-graduação em Informática do Departamento de Informática da PUC-Rio como requisito parcial para obtenção Do título de Doutor em Informática

Orientador: Prof. Carlos José Pereira de Lucena 


\title{
Baldoino Fonsêca dos Santos Neto
}

\author{
Uma Abordagem Deontica para o \\ Desenvolvimento de Agentes Normativos \\ Autônomos
}

Tese apresentada ao Programa de Pós-graduação em Informática do Departamento de Informática do Centro Técnico Científico da PUC-Rio como requisito parcial para obtenção Do título de Doutor em Informática. Aprovada pela Comissão Examinadora abaixo assinada.

\author{
Prof. Carlos José Pereira de Lucena \\ Orientador \\ Departamento de Informática - PUC-Rio
}

Prof. Hugo Fuks

Departamento de Informática - PUC-Rio

Prof. Simone Diniz Junqueira Barbosa

Departamento de Informática - PUC-Rio

Prof. Viviane Torres da Silva Universidade Federal Fluminense - UFF

Prof. Paulo Sérgio Conceição de Alencar

University of Waterloo

Prof. José Eugenio Leal

Coordenador Setorial do Centro Técnico Científico - PUC-Rio 
Todos os direitos reservados. É proibida a reprodução total ou parcial do trabalho sem autorização da universidade, do autor e do orientador.

Baldoino Fonsêca dos Santos Neto

Obteve seu Mestrado na PUC-Rio em 2010. Atualmente atua na área de Desenvolvimento de Software Orientado a Agentes no Laboratório de Engenharia de Software (LES) da PUCRio.

Ficha Catalográfica

Neto, Baldoino Fonsêca dos Santos

Uma Abordagem Deontica para o Desenvolvimento de Agentes Normativos Autônomos / Baldoino Fonsêca dos Santos Neto; orientador: Carlos José Pereira de Lucena. - Rio de Janeiro: PUC-Rio, Departamento de Informática, 2012.

169 f. ; $30 \mathrm{~cm}$

1. Tese (doutorado) - Pontifícia Universidade Católica do Rio de Janeiro, Rio de Janeiro, 2010.

Inclui referências bibliográficas.

1. Informática - Tese. 2. Agentes Autônomos. 3. Mecanismos Normativos. 4. Arquitetura de Software. 5. Linguagem de Programação.

I. Lucena, Carlos. II. Pontifícia Universidade Católica do Rio de Janeiro. Departamento de Informática. III. Título. 


\section{Agradecimentos}

Primeiramente, agradeço a Deus, por minha existência, por me conceder momentos maravilhosos. A minha família querida, meu pai José Pedro (In memorian). A minha mãe Tercília, sempre preocupada com o meu bem-estar e esforçando-se ao máximo para me dar uma educação promissora. Ao meu irmão Walfran, pelo o seu apoio espiritual, companheiro da minha fortaleza em toda essa jornada de lutas árduas. As minhas imãs Walflania e Walfraneide. A minha namorada Bárbara, você é a peça chave da minha vida. Esta vitória é nossa. A Dona Auxiliadora é uma satisfação, dividir esta conquista com a senhora. Obrigado pelo carinho e atenção. Professor Carlos Lucena, meu orientador, com quem eu tive a oportunidade de aprender e interagir, tornando o trabalho mais valioso e gratificante. Professora Viviane Silva, a quem admiro, todo o seu empenho e dedicação. Aos colegas Elder e Manoel, meus parceiros de trabalho desde o início do doutorado. Aos amigos do LES, que muito contribuíram para o meu avanço profissional. Ao departamento de informática, professores e secretaria Por fim, a CNPq e PUC-Rio, pela ajuda financeira. 


\section{Resumo}

Neto, Baldoino Fonsêca dos Santos; Lucena, Carlos José Pereira de (Orientador). Uma Abordagem Deontica para o Desenvolvimento de Agentes Normativos Autônomos. Rio de Janeiro, 2012. 169p. Tese de Doutorado - Departamento de Informática, Pontifícia Universidade Católica do Rio de Janeiro.

Normas sociais têm se tornado uma das abordagens mais promissoras para garantir uma ordem social desejável em sistemas multiagentes. Nestes sistemas, agentes autônomos trabalham a fim de atingir objetivos comuns ou diferentes. Normas regulam o comportamento de tais agentes definindo obrigações e proibições, fornecendo recompensas e estabelecendo punições a fim de incentivá-los a se comportarem de acordo com as normas. Embora o uso de normas seja um mecanismo promissor para regular o comportamento de agentes, o desenvolvimento de agentes capazes de atuar em um sistema, adotar as normas e lidar com as mesmas autonomamente tem sido mais difícil do que o desenvolvimento de agentes tradicionais. A razão para esta dificuldade é a falta de mecanismos que possibilitem o desenvolvimento de agentes autônomos capazes de lidar com questões relacionadas a normas. Nesta tese, apresenta-se uma abordagem para desenvolvimento de agentes capazes de lidar autonomamente com questões normativas. Para tanto, a abordagem fornece um modelo arquitetural para apoiar um agente no raciocínio sobre as normas. Tal modelo estende o modelo Belief-Desire-Intention adicionando um conjunto de funções que auxiliam o agente na adoção de novas normas, na verificação da ativação, desativação, cumprimento e violação das normas, na seleção de quais normas devem ser cumpridas ou violadas, na detecção e resolução de conflitos entre normas, na geração de novos objetivos e na seleção de objetivos, planos e intenções, levando em consideração as normas do sistema. Além disto, a abordagem fornece os mecanismos necessários para implementação de agentes projetados a partir do modelo arquitetural proposto. Dois cenários são utilizados para exemplificar a abordagem proposta. O primeiro está relacionado ao planejamento de missões de resgate reguladas por normas. O segundo está relacionado ao suporte ao desenvolvimento de software regulado por normas. Por fim, experimentos são apresentados que demonstram a importância de desenvolver agentes a partir da abordagem proposta seja na visão do agente ou da sociedade.

\section{Palavras-chave}

Agentes Autônomos; Mecanismos Normativos; Arquitetura de Software; Linguagem de Programação; 


\section{Abstract}

Neto, Baldoino Fonsêca dos Santos; Lucena, Carlos José Pereira de (Advisor). A Deontic Approach to Develop Autonomous Normative Agents. Rio de Janeiro, 2012. 169p. Doctoral Thesis - Departamento de Informática, Pontifícia Universidade Católica do Rio de Janeiro.

Social norms have become one of the most promising approaches toward ensuring a desirable social outcome in multi-agent systems. In these systems, autonomous and heterogeneous agents work toward common or different goals. Norms regulate the behaviour of these agents by defining obligations and prohibitions, and by creating rewards and penalties to encourage the agents to behave so as to meet these norms. Although the use of norms is a promising mechanism to regulate the agents' behavior, the development of agents able to join an agents society, to adopt the norms and to work with them autonomously has shown to be significantly more challenging than traditional agents. The reason for this difficulty is the lack of agent models that allow developing of autonomous agents able to cope with issues related to norms. In this thesis, we introduce an approach to develop agents able to deal with normative questions in an autonomous way. The approach extends the Belief-DesireIntention model including functions that assist the agent in adopting new norms, in verifying norm activation, deactivation, fulfillment and violation, in selecting the norms to be fulfilled or violated by the agent, in identifying and overcoming conflicts among norms, in generating new goals from norms and in selecting goals, plans and intentions while taking into account the system norms. In addition, the approach provides mechanisms that are necessary to implement agents designed from the proposed architectural model. Two scenarios are used in order to exemplify the proposed approach. The first is related to the planning of rescue missions regulated by norms. The second is related to the support to software development regulated by norms. Finally, experiments that demonstrate the importance of developing agent by using the proposed architectural model, both in the agent and society point of view, are presented.

\section{Keywords}

Autonomous Agents; Normative Mechanisms; Software Architecture; Programming Language; 


\section{Sumário}

1 Introdução $\quad 11$

$\begin{array}{lll}1.1 & \text { Problema } & 12\end{array}$

$\begin{array}{lll}1.2 & \text { Limitações das Abordagens Atuais } & 13\end{array}$

$\begin{array}{lll}1.3 & \text { Questões de Pesquisa } & 13\end{array}$

$\begin{array}{lll}1.4 & \text { Solução Proposta } & 14\end{array}$

$\begin{array}{ll}1.5 \text { Contribuições } & 15\end{array}$

1.6 Organização da Tese 15

2 Fundamentação Teórica $\quad 17$

$\begin{array}{lll}2.1 & \text { Sistemas Multiagentes } & 17\end{array}$

$\begin{array}{lll}2.2 & \text { Arquitetura BDI } & 19\end{array}$

2.3 AgentSpeak(L) 20

2.4 Interpretador Jason 23

2.5 Normas e Agentes Normativos 24

2.6 Linguagem de Especificação Formal Z 25

$\begin{array}{lll}2.7 & \text { Considerações Finais } & 29\end{array}$

3 Trabalhos Relacionados $\quad 30$

3.1 Agentes Simples 30

3.2 Agentes Interessados 32

3.3 Considerações Finais 33

4 Arquitetura $\quad 34$

4.1 Tipos 34

4.1.1 Comportamentos 36

4.1.2 Normas 39

$\begin{array}{lll}4.1 .3 & \text { Planos } & 44\end{array}$

4.1.4 Intenções 46

$\begin{array}{lll}4.2 & \text { Estrutura ANA } & 46\end{array}$

4.3 Visão Operacional de ANA 49

4.3.1 Beliefs and Norms Reviewer $\quad 50$

4.3.2 Desires Normative Generator $\quad 55$

4.3.3 Normative Filter 64

4.3.4 Intention Normative Executor and Selector $\quad 75$

$\begin{array}{lll}4.4 & \text { Considerações Finais } & 79\end{array}$

5 Implementando ANA $\quad \mathbf{8 0}$

$\begin{array}{lll}5.1 & \text { AgentSpeak(L) Normativo } & 80\end{array}$

5.2 Interpretador Jason Normativo 83

5.2.1 Função Normative Reviewer 85

5.2.2 Função Events Normative Generator 88

$\begin{array}{ll}5.2 .3 \text { Selecting Event } & 90\end{array}$

$\begin{array}{lll}\text { 5.2.4 Check Context } & 91\end{array}$

$\begin{array}{ll}\text { 5.2.5 Selecting Plan } & 94\end{array}$ 
$\begin{array}{lll}\text { 5.2.6 Review Intention } & 96\end{array}$

5.2.7 Selecting Intention 98

5.2.8 Execute Intention 98

$\begin{array}{ll}\text { 5.2.9 Considerações Finais } & 100\end{array}$

6 Cenários 102

6.1 Cenário 1: Agentes de Suporte para Planejamento de Missões de Resgate Reguladas por Normas 102

6.1.1 Representação de Objetivos, Ações, Planos e Normas 106

6.1.2 Adotando Novas Normas 113

6.1.3 Revisando as Normas Ativadas e Desativadas 114

6.1.4 Verificando as Normas Cumpridas ou Violadas 114

6.1.5 Detectando e Superando Conflitos Normativos 115

6.1.6 Selecionando Normas para serem Cumpridas ou Violadas 115

6.1.7 Aplicando Decisões Normativas 116

$\begin{array}{ll}\text { 6.1.8 Seleção de Eventos } & 116\end{array}$

6.1.9 Verificando Planos Relevantes e Aplicáveis 119

6.1.10 Seleção de Planos 119

6.1.11 Revisão de Intenções 122

6.1.12 Execução e Monitoramento de Intenções 124

6.2 Cenário 2: Desenvolvimento e Evolução de Software Suportado por Agentes Regulados por Normas 124

6.2.1 Representação de Objetivos, Ações, Planos e Normas 129

6.2.2 Adotando Novas Normas 134

6.2.3 Revisando as Normas Ativadas e Desativadas 135

6.2.4 Verificando as Normas Cumpridas ou Violadas 135

6.2.5 Detectando e Superando Conflitos entre Normas 135

6.2.6 Selecionando Normas para serem Cumpridas ou Violadas 136

6.2.7 Aplicando Decisões Normativas 137

$\begin{array}{ll}\text { 6.2.8 Seleção de Eventos } & 138\end{array}$

6.2.9 Verificando Planos Relevantes e Aplicáveis 141

6.2.10 Seleção de Planos 141

6.2.11 Revisão de Intenções 143

6.2.12 Execução e Monitoramento de Intenções 144

$\begin{array}{ll}6.3 \text { Considerações Finais } & 144\end{array}$

7 Experimento $\quad 145$

$\begin{array}{lll}7.1 & \text { Fatores de Comparação } & 146\end{array}$

$\begin{array}{lll}\text { 7.1.1 Estratégias de Deliberação de Normas } & 146\end{array}$

$\begin{array}{ll}\text { 7.1.2 Efetivando as Decisões Normativas } & 150\end{array}$

$\begin{array}{ll}\text { 7.1.3 Processo de Seleção } & 151\end{array}$

$\begin{array}{lll}7.1 .4 & \text { Análise Qualitativa } & 153\end{array}$

7.2 Configuração do Experimento 153

$\begin{array}{lll}7.2 .1 \text { Bases } & 153\end{array}$

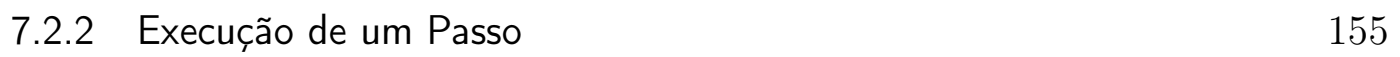

$\begin{array}{lll}\text { 7.2.3 Registrando as Informações } & 156\end{array}$

$\begin{array}{ll}\text { 7.2.4 Parâmetros para Avaliação } & 156\end{array}$

$\begin{array}{lll}7.3 & \text { Resultados do Experimento } & 156\end{array}$

$\begin{array}{lll}7.4 & \text { Considerações Finais } & 158\end{array}$ 
8 Conclusão e trabalhos futuros

159

8.1 Limitações do Trabalho

160

8.2 Trabalhos Futuros 


\section{Lista de figuras}

2.1 Arquitetura BDI (reproduzida de (Weiss 1999)) 19

2.2 Sistema de Raciocínio Procedural (reproduzida de (Bordini et al. 2007) ) 20

2.3 Interpretador Jason 23

$\begin{array}{lll}\text { 4.1 Arquitetura para projetar ANA } & 49\end{array}$

5.1 Jason Normativo 83

$\begin{array}{lll}7.1 & \text { Resultados } & 157\end{array}$ 